Chronic Obstructive Pulmonary Diseases:

Journal of the COPD Foundation

\author{
Original Research
}

\title{
Effectiveness of Umeclidinium-Vilanterol for Protocolized Management of Chronic Obstructive Pulmonary Disease Exacerbation in Hospitalized Patients: A Sequential Period Analysis
}

Todd W. Chapin, PharmD, BCPS ${ }^{1}$ Michael A. Mann, MPH $^{2}$ Gary L. Brown, RRT, FAARC ${ }^{3}$

Traci L. Leitheiser, BS, RRT, CTTS ${ }^{3}$ Becky Anderson, RRT ${ }^{3}$ David D. Leedahl, PharmD, BCPS-AQ ID, BCCCP ${ }^{1}$

\section{Abstract}

Background: Bronchodilator therapy is a foundation of chronic obstructive pulmonary disease (COPD) exacerbation treatment. Although international guidelines recommend short-acting formulations given multiple times per day, long-acting formulations have not been adequately evaluated. The objective of our study was to determine the effectiveness of umeclidinium-vilanterol (UME/VIL), long-acting beta2-agonist/long-acting muscarinic antagonist (LABA/LAMA) as a once-daily alternative for treating COPD exacerbations in hospitalized patients.

Methods: In this retrospective sequential period analysis, we reviewed electronic medical records of patients hospitalized for COPD exacerbations before (September 1, 2015 to February 29, 2016) and after (April 1, 2016 to September 30, 2016) incorporation of UME/VIL into our standard COPD protocol. Before implementation, patients received a daily anticholinergic plus twice-daily long-acting beta2-agonist therapy (tiotropium plus formoterol, $n=65)$. After implementation, UME/VIL replaced the previous regimen $(n=58)$. No other changes were made to the COPD protocol. The primary outcome was 30-day hospital readmission rate. Hospital length of stay, 30-day mortality, and cost of care were analyzed as secondary outcomes.

Results: A trend toward increased 30-day readmission rates in the post-intervention group (24.1\% versus $10.8 \%$, $p=0.049$ ) was no longer statistically significant after adjustment for severity of illness (based on case-mix index) and complications or comorbidities based on diagnosis-related group codes (adjusted odds ratio: 2.499; 95\% confidence interval: 0.916-7.380; $p=0.074$ ).

Conclusion: After adjustment for potential confounders, the implementation of a LABA/LAMA combination product was not statistically associated with an increased 30-day readmission rate but was associated with lower cost of care.

\begin{abstract}
Abbreviations: chronic obstructive pulmonary disease, COPD; umeclidinium/vilanterol, UME/VIL; long-acting beta2-agonist/long-acting muscarinic antagonist, LABA/LAMA; pulmonary function test, PFT; forced expiratory volume in 1 second, FEV $\mathbf{1}$; forced vital capacity, FVC; Global initiative for chronic Obstructive Lung Disease, GOLD; electronic medical record, EMR; Medicare severity diagnosis-related groups, MS-DRG; case-mix index, CMI; Center for Disease Control, CDC; International Classification of Disease(s), ICD; standard deviation, SD Funding Support: None

Date of Acceptance: October 24, 2017

Citation: Chapin TW, Mann MA, Brown GL, Leitheiser TL, Anderson B, Leedahl DD. Effectiveness of umeclidinium-vilanterol for protocolized management of chronic obstructive pulmonary disease exacerbation in hospitalized patients: A sequential period analysis. Chronic Obstr Pulm Dis. 2018;5(1):38-45. doi: https://doi.org/10.15326/jcopdf.5.1.2017.0163
\end{abstract}

\section{This article contains an online supplement.}

1 Pharmacy Services, Sanford Health, Fargo, North Dakota
2 North Dakota State University College of Health Professions, Fargo

3 Respiratory Care Services, Sanford Health, Fargo, North Dakota 


\section{Address correspondence to:}

David D. Leedahl, PharmD

801 Broadway N, Rt 0204

Fargo, ND 58122

Phone: (701) 417-2309

E-mail: david.leedahl@sanfordhealth.org

\section{Keywords:}

chronic obstructive pulmonary disease; COPD; exacerbations; bronchodilator; umeclidinium; vilanterol; tiotropium; formoterol; hospital readmission; length of stay; treatment protocol; respiratory therapy

\section{Introduction}

Chronic obstructive pulmonary disease (COPD) is the $4^{\text {th }}$ leading cause of mortality worldwide, accounting for 3 million deaths annually. It also affects more than $6 \%$ of the United States population. ${ }^{1-3}$ Characterized by persistent, non-reversible airflow limitations and progressive inflammatory response within the airways to gasses or noxious particles, COPD is often diagnosed utilizing pulmonary function tests (PFTs), specifically the ratio of forced expiratory volume in 1 second $\left(\mathrm{FEV}_{1}\right)$ to forced vital capacity (FVC) of less than 0.7 and a predicted $\mathrm{FEV}_{1}$ less than $80 \%$.

Acute changes from a patient's baseline, such as dyspnea or a cough, beyond the patient's normal daily variability lead physicians to diagnose COPD exacerbations, often requiring medical intervention. ${ }^{4}$ Inhaled bronchodilators are a cornerstone of treatment and prevention of COPD exacerbations, although they are often associated with multiple doses per day and significant drug and administration cost during exacerbation management, often leading to poor adherence. ${ }^{5-7}$ In addition to medicationrelated expenses, COPD exacerbations may also lead to untoward emergency department or office visits, hospitalizations, and days missed from work. Indeed, COPD exacerbations account for the largest portion of costs for COPD health care. ${ }^{8}$ Health systems are also increasingly scrutinized for unplanned hospital readmissions after exacerbation treatment and may face decreased reimbursement. ${ }^{9}$

The burdens of optimized medication management are counterbalanced by the benefits of improved lung function and exercise tolerance, reduced exacerbation frequency and severity, and prolonged survival. ${ }^{5,10-12}$ While there are several well-monitored efficacy trials and meta-analyses in treating COPD, they often have limited inclusion/exclusion criteria, so they may have limited applicability to everyday patients. The 2017 Global initiative for chronic Obstructive Lung Disease (GOLD) guidelines ${ }^{5}$ do not specifically address the advantages of long-acting combination products used in treatment of COPD exacerbations. Lastly, there are few studies highlighting the advantages of a respiratory therapist-driven protocol for exacerbation treatment. Therefore, in this study, we sought to compare outcomes utilizing umeclidinium-vilanterol (UME/VIL) in clinical practice on hospitalized patients using a standardized protocol driven therapy for COPD exacerbations.

\section{Methods}

\section{Study Design and Assessments}

Our 583-bed tertiary care hospital utilizes a physician-initiated, respiratory therapist-managed treatment protocol for COPD exacerbations (online supplementary data). The long-acting beta2-agonist/ long-acting muscarinic antagonist (LABA/LAMA) medication regimen within this protocol (tiotropium and formoterol) was changed on March 15, 2016 to a combination product, UME/VIL, due to the withdrawal of formoterol fumarate inhalation powder from the market in the United States. This medication substitution was the only change made to the protocol during the study periods. We performed a retrospective, sequential period analysis during the timeframe of September 2015 through September 2016 comparing 30-day readmission rates, with March 2016 serving as the washout period. The secondary outcomes of 30day mortality, length of hospital stay, and cost of care were also collected for these study periods. The study was approved by the institutional review board with a waiver of informed consent and Health Insurance Portability and Accountability Act authorization and was performed in accordance with the ethical standards of the 1964 Declaration of Helsinki and its later amendments.

\section{Study Patient Population}

Electronic medical records (EMRs) were identified for consecutive patients coded for acute exacerbation of COPD utilizing Medicare severity diagnosis-related groups (MS-DRGs) 190-192, based on physician diagnosis. We did not evaluate 47 patients admitted during the 1-month washout period in order to avoid the potential confounding variable of a patient receiving medications from both protocols while hospitalized. Therefore, the pre-implementation group included 
patients admitted from September 1, 2015 to February 29, 2016, $(n=65)$, and the post-implementation group included patients admitted from April 1, 2016 to September 30, 2016, $(n=58)$.

The COPD protocol was only implemented for adult patients with inspiratory flow measurement values $>40 \mathrm{~L} / \mathrm{min}$ (utilizing In-Check ${ }^{\mathrm{TM}}$ inspiratory flow measurement device, Alliance Tech Medical, Granbury, Texas). Therefore, only patients meeting these criteria during their index hospitalization during each study timeframe were eligible for inclusion.

\section{Data Elements and Definitions}

In addition to 30-day mortality, readmission rates, hospital length of stay, and cost of care, the following information was collected for each patient: demographics (age, race, gender, weight, body mass index), home oxygen use, smoking status, and associated pack years. A complete summary of baseline characteristics collected for each patient is provided in Table 1. PFT results were recorded for each patient and reflected the most recent results prior to admission unless PFT results were available after admission within a shorter timeframe. The severity of illness for each patient was assessed using case-mix index (CMI), initial bronchodilator therapy assessment score, (see the COPD treatment protocol in the online data supplement for score calculation) and presence of complications and comorbidities based on the MS-DRG utilized for third party reimbursement. Within the MS-DRG categorization, the COPD exacerbation diagnosis was categorized as one of the following: without complications or comorbidities, with complications and comorbidities, or with major complications and comorbidities. We also collected the total daily direct costs of the admission as well as respiratory therapy and pharmacy direct costs per day. All costs were determined by EMR charge reports.

Influenza vaccination status was considered up to date if an influenza vaccination was documented prior to admission during an influenza season, defined by the Centers for Disease Control and Prevention (CDC) as October 1- March 31. Outside of influenza season, a patient was considered vaccinated against influenza if an influenza vaccination was documented during the most recent influenza season. A patient was considered vaccinated for pneumonia if they had received any formulation of the pneumococcal vaccine within the last 5 years, or if they had no indication to receive it per CDC guidelines (indications include patients $>65$ years of age; cigarette smokers; patients with asthma, diabetes, alcoholism, chronic lung, liver, heart, or kidney illnesses, weakened immunity, cochlear implants, or cerebrospinal fluid leaks). ${ }^{13}$ The use of corticosteroids and anti-infective medications was determined by the presence of these medications on the participant's outpatient medication list prior to admission to the hospital. Baseline characteristics related to comorbidities were determined by International Classification of Disease(s) (ICD)-9 or ICD-10 codes associated with those conditions (online data supplement).

\section{Statistical Analysis}

Pearson's Chi-squared tests were used for categorical variables. T-tests and Wilcoxon rank-sum tests were used for the statistical analysis of parametric and nonparametric continuous variables, respectively. Data are presented as percentages with the mean \pm standard deviation (SD), or the median and interquartile range when appropriate. Data were initially assessed by univariable analysis, and variables associated with and clinically relevant to the dependent outcomes of 30day hospital readmissions, 30-day mortality, hospital length of stay, and cost of care with a $p$-value $\leq 0.05$ were included in the multivariable analysis to adjust the analysis for potential confounding variables. Data were analyzed with JMP software, version 12.2.0 (Cary, North Carolina).

\section{Results}

We reviewed 123 patients hospitalized for COPD exacerbation (65 in the pre-intervention cohort, 58 in the post-intervention group). Baseline characteristics between groups were similar (Table 1). Univariable analysis demonstrated that the post-intervention group who received the UME/VIL-based protocol was statistically associated with a higher 30-day readmission rate $(24.14 \%$ versus $10.77 \%, p=0.049$, Table 2). However, the UME/VIL group had a statistically higher rate of major complications or comorbidities (MS-DRG 190, $72.4 \%$ versus $44.6 \%$, $p=<0.01$ ) as well as a higher average CMI (1.06 versus 0.99, $p=0.029$ ). We reviewed the average CMI for all hospitalized patients during the study periods to determine whether the increase in CMI was limited to the COPD patients in our study, or was a trend seen throughout the hospital. We determined that the 


\section{Table 1. Characteristics of Patients Hospitalized for Chronic Obstructive Pulmonary Disease Exacerbation}

\begin{tabular}{|c|c|c|c|}
\hline & $\begin{array}{l}\text { Pre-intervention } \\
\qquad(n=65)\end{array}$ & $\begin{array}{l}\text { Post-intervention } \\
\qquad(n=58)\end{array}$ & $P$ value \\
\hline Sex (male) & 29 (44.6) & $29(50.0)$ & 0.550 \\
\hline Age (years) & $69.0(12.7)$ & $69.2(10.3)$ & 0.925 \\
\hline \multicolumn{4}{|l|}{ Age Group } \\
\hline$<50$ & $4(6.2)$ & $2(3.5)$ & 0.487 \\
\hline $50-59$ & $13(20.0)$ & $10(17.2)$ & 0.695 \\
\hline $60-69$ & $16(24.6)$ & $13(22.4)$ & 0.774 \\
\hline $70-79$ & 17 (26.2) & $23(39.7)$ & 0.111 \\
\hline$>80$ & $15(23.1)$ & $10(17.2)$ & 0.422 \\
\hline \multicolumn{4}{|l|}{ Race/Ethnicity } \\
\hline White & $60(92.3)$ & $54(93.1)$ & 0.866 \\
\hline Black & $0(0.0)$ & $2(3.5)$ & 0.131 \\
\hline American Indian/Alaskan & $4(6.2)$ & $2(3.5)$ & 0.487 \\
\hline Asian & $1(1.5)$ & $0(0)$ & 0.343 \\
\hline Weight $(\mathrm{kg})$ & $75.4(26.6)$ & $78.4(25.0)$ & 0.527 \\
\hline Body Mass Index, $\mathrm{kg} / \mathrm{m}^{2}$ & $26.6(8.6)$ & $27.9(9.0)$ & 0.432 \\
\hline Pack Years of Current/Former Smokers, years & $37.7(24.6)$ & $39.8(31.1)$ & 0.737 \\
\hline \multicolumn{4}{|l|}{ Smoking Status } \\
\hline Current smoker & $26(40.0)$ & $21(36.2)$ & 0.666 \\
\hline Former smoker & $36(55.4)$ & $35(60.3)$ & 0.578 \\
\hline Never smoked & $3(4.6)$ & $2(3.5)$ & 0.744 \\
\hline Long-term Home Oxygen Usage & $42(64.6)$ & $37(63.8)$ & 0.924 \\
\hline Case-mix Index & $0.99(0.16)$ & $1.06(0.16)$ & 0.029 \\
\hline \multicolumn{4}{|l|}{ Medicare Severity Diagnosis-Related Group (MS-DRG) } \\
\hline COPD exacerbation with MCC (MS-DRG 190) & 29 (44.6) & $42(72.4)$ & 0.002 \\
\hline COPD exacerbation with CC (MS-DRG 191) & $28(43.1)$ & $9(15.5)$ & $<0.001$ \\
\hline COPD exacerbation without MCC or CC (MS-DRG 192) & $8(12.3)$ & $7(12.1)$ & 0.968 \\
\hline \multicolumn{4}{|l|}{ Pulmonary Function Test Results ${ }^{\mathbf{a}}$} \\
\hline FVC Pre & $2.31(0.73)$ & $2.47(0.70)$ & 0.251 \\
\hline FVC \% Pre Predicted & $65.92(15.49)$ & $71.16(18.83)$ & 0.122 \\
\hline FVC Post & $2.39(0.74)$ & $2.65(0.82)$ & 0.186 \\
\hline FEV 1 Pre & $1.17(0.49)$ & $1.25(0.51)$ & 0.434 \\
\hline FEV $1 \%$ Pre Predicted & $44.42(16.92)$ & $48.06(17.40)$ & 0.276 \\
\hline FEV 1 Post & $1.22(0.53)$ & $1.39(0.50)$ & 0.195 \\
\hline FEV 1 /FVC Pre & $51.20(15.65)$ & $50.31(12.64)$ & 0.742 \\
\hline FEV 1 /FVC Post & $50.58(12.86)$ & $52.12(11.16)$ & 0.610 \\
\hline Initial Bronchodilator Therapy Assessment Score & $2.30(1.59)$ & $2.44(1.61)$ & 0.641 \\
\hline Short-acting Beta2-Agonist doses per day ${ }^{b}$ & $0.32(0.55)$ & $0.38(0.72)$ & 0.619 \\
\hline \multicolumn{4}{|l|}{ Comorbid Conditions } \\
\hline Asthma & $13(20.0)$ & $11(19.0)$ & 0.885 \\
\hline Diabetes & $43(66.2)$ & $36(62.1)$ & 0.637 \\
\hline Acute coronary syndrome & $24(36.9)$ & $17(29.3)$ & 0.371 \\
\hline \multicolumn{4}{|l|}{ Vaccination Status } \\
\hline Influenza & $38(58.5)$ & $35(60.3)$ & 0.832 \\
\hline Pneumococcal & $55(84.6)$ & $50(86.2)$ & 0.803 \\
\hline \multicolumn{4}{|l|}{ Concomitant Medications } \\
\hline Anti-infective agents & $38(58.5)$ & $33(56.9)$ & 0.861 \\
\hline Corticosteroid agents & $36(55.4)$ & $28(48.3)$ & 0.431 \\
\hline \multicolumn{4}{|l|}{ Payer } \\
\hline Medicare & 48 (73.9) & $42(72.4)$ & 0.858 \\
\hline Medicaid & $9(13.9)$ & $4(6.9)$ & 0.211 \\
\hline Private insurance & $6(9.2)$ & $10(17.2)$ & 0.187 \\
\hline Self & $1(1.5)$ & $2(3.5)$ & 0.493 \\
\hline Veterans affairs & 1 (1.5) & $0(0)$ & 0.343 \\
\hline
\end{tabular}

Data are mean (standard deviation) or $\mathrm{n}(\%)$

$\mathrm{kg}=$ kilogram; $\mathrm{kg} / \mathrm{m}^{2}=$ kilogram per meter squared; $\mathrm{MS}-\mathrm{DRG}=$ Medicare severity diagnosis-related groups; $\mathrm{MCC}=$ major complications / comorbidities; $\mathrm{CC}=$ complications / comorbidities; $\mathrm{FVC}=$ forced vital capacity; $\mathrm{FEV}_{1}=$ forced expiratory volume in 1 second

a $87.8 \%$ of patients had recorded PFT results, $52.8 \%$ patients had recorded post PFT results. Data are liters (standard deviation) for volume or \% (standard deviation) for predicted volume

bAlbuterol and levalbuterol 
medical center's average CMI (excluding newborns) was 1.65 and 1.70 in the pre- and post-intervention periods, respectively, indicating that the increase in CMI was not limited to COPD patients at our facility.

After inclusion of the statistically significant variables of CMI and MS-DRG 190 into a multiple logistic regression model, the association between the post-intervention UME/VIL group and 30-day readmission was no longer statistically significant (adjusted odds ratio, 2.50; 95\% confidence interval, 0.92-7.38).

Differences in 30-day mortality and hospital length of stay were not statistically significant (Table 2). The overall mean (SD) direct cost per patient-day decreased from $\$ 968.34$ (176.92) to $\$ 903.09$ (221.36) but was not statistically significant $(p=0.076)$. Respiratory therapyassociated mean (SD) daily direct cost significantly decreased from $\$ 116.04$ to $\$ 90.77$ (63.10 and 66.03 respectively, $p=0.033$ ). We noted a numerical decrease in pharmacy-associated mean (SD) direct daily cost from $\$ 79.66$ (32.88) to $\$ 72.26$ (30.71). While it did not reach statistical significance $(p=0.199)$, the impact of reducing daily cost by $\$ 7.40$ per patient may be financially significant. For a complete cost analysis, see Table 3.

\section{Discussion}

While the advantages of once daily combination medications are intuitive regarding adherence and convenience in the prevention of COPD exacerbations, there are few studies comparing these products in patients hospitalized for an exacerbation. The most recent GOLD guidelines suggest that utilizing bronchodilators with multiple mechanisms of action may be more effective than single medications, at lower doses that may reduce side effects, but they do not suggest that combination products have specific benefits over using multiple separate medications. It is reasonable that reducing the number of daily administrations and medication expense would be appropriate, assuming there is no correlative increase in adverse patient outcomes.

While we noted an increased rate of readmissions in the UME/VIL study group, it is difficult to ascertain whether they are attributable to the medication regimen change of UME/VIL replacing tiotropium and formoterol, the higher severity of illness found during the second study period, or other unmeasured confounding variables. Multiple logistic regression analysis of outcomes, however, indicated that the

\section{Table 2. Association of Umeclidinium/Vilanterol-Based Protocol with Clinical Outcomes}

\begin{tabular}{|c|c|c|c|}
\hline & $\begin{array}{l}\text { Pre-intervention } \\
\qquad(\mathrm{n}=65)\end{array}$ & $\begin{array}{l}\text { Post-intervention } \\
\quad(n=58)\end{array}$ & $P$ value \\
\hline 30-Day Readmission Rates & $7(10.77)$ & $14(24.14)$ & 0.049 \\
\hline $\begin{array}{l}\text { 30-Day Readmission Model Adjusted for Medicare Severity } \\
\text { Diagnosis-Related Groups, Case-Mix Index }\end{array}$ & \multirow{2}{*}{\multicolumn{2}{|c|}{$\begin{array}{r}\text { Odds ratio 2.499, } \\
95 \% \text { confidence interval (0.92-7.38) }\end{array}$}} & 0.074 \\
\hline Hospital Length of Stay (days) & & $5.05(4.25)$ & 0.300 \\
\hline 30-day Mortality & $3(4.62)$ & $3(5.17)$ & 0.886 \\
\hline
\end{tabular}

Data are mean (standard deviation) or $\mathrm{n}(\%)$

\section{Table 3. Direct Cost Associated with Chronic Obstructive Pulmonary Disease Exacerbation (\$US)}

\begin{tabular}{l|rrr} 
& \multicolumn{3}{l}{$\begin{array}{c}\text { Pre-intervention } \\
\text { Post-intervention } P \text { value }\end{array}$} \\
\hline Respiratory Therapy Direct Cost & $(\mathrm{n}=\mathbf{6 5})$ & \multicolumn{1}{c}{$(\mathrm{n}=58)$} & \\
\hline Respiratory therapy Direct Cost per day & $494.27(427.43)$ & $463.44(433.83)$ & 0.693 \\
\hline Pharmacy Direct Cost & $116.04(63.10)$ & $90.77(66.03)$ & 0.033 \\
\hline Pharmacy Direct Cost per day & $352.44(371.05)$ & $347.78(285.17)$ & 0.938 \\
\hline Total Direct Cost & $79.66(32.88)$ & $72.26(30.71)$ & 0.199 \\
\hline Total Direct Cost per day & $4106.67(2955.91)$ & $4429.77(3619.09)$ & 0.591 \\
\hline Day & $968.34(176.92)$ & $903.09(221.36)$ & 0.076 \\
\hline
\end{tabular}

Data are mean (standard deviation) 
medication was not independently associated with increased readmissions (when adjusted for severity of illness). Patients admitted during the 2 study periods exhibited similar characteristics. Patients in both study periods had comparable demographic backgrounds, as well as similar rates of home oxygen use and influenza and pneumococcal vaccinations, indicating well-matched study populations. We found no statistically significant difference in the number of short-acting beta2-agonist (albuterol and levalbuterol) administrations per day for patients in the pre- and post-study populations. Smoking is a critical risk factor for COPD exacerbations, as patients who continue to smoke experience accelerated decline in $\mathrm{FEV}_{1}$ when compared to nonsmokers. ${ }^{14}$ While the EMR review did not yield complete smoking history, the data that were lacking were evenly distributed between study populations. We obtained pack year history from 41 and 37 patients respectively in pre- and post-study periods (66.1\% of current or former smokers in each study population). A similar distribution of patients identified as current, former, or never smokers was found in each cohort (Table 1).

The diagnosis of COPD is based on spirometry measurements, requiring the presence of postbronchodilator $\mathrm{FEV}_{1} / \mathrm{FVC}$ of $<0.7$ for confirmation. ${ }^{5}$ In patients with COPD exacerbations and acutely compromised pulmonary function, performing PFTs at the time of admission to confirm a diagnosis of COPD is controversial. ${ }^{15}$ In our study, we relied on MS-DRG coding to identify our patient cohort, which may have not identified all eligible patients for inclusion into our study. Indeed, we were able to collect pre-bronchodilator spirometry data on 108 of 123 patients (59 and 49 in pre- and post-groups, respectively), however, only 65 patients had both pre- and post-bronchodilator results documented in our EMRs (53\% of the study cohort, 31 and 34 in pre- and post-groups, respectively). Out of those 65 with post-bronchodilator data, all but 2 patients had a documented FEV 1 /FVC in their medical record consistent with COPD. In the cohort of patients with any evaluable PFT data, we found no statistical differences between the pre- and post-groups.

Our facility has an advantage in comparing 2 COPD treatment regimens, as while the diagnosis of and decision to treat a COPD exacerbation is at the discretion of a physician, our respiratory therapists follow a rigid protocol and we, therefore, experience minimal deviation in the course of therapy between similar patients. This standardized therapy minimizes the potential for bias or variations in treatment between physicians as well as reducing the possibility of changing prescription habits over time.

Several limitations of our study should be noted. Our study was conducted at a single center and was retrospective in nature, and our control group was a historical cohort. As a single-center study utilizing EMR review, we did not have the ability to record certain patient events such as vaccinations occurring at other health care systems or outcomes such as readmissions to medical centers not sharing our EMR system. We relied on diagnostic (MS-DRG) coding which is, at times, imprecise and variable. ${ }^{16}$ Seasonal variability such as influenza season and weather may play a role in COPD exacerbation admission rates or severity. The pre-intervention study period partially coincided with the influenza reporting season, defined by the CDC as October 1 through March 31. ${ }^{17}$ An analysis of the TORCH study found higher COPD exacerbation and hospitalization rates in winter months in northern climates such as ours, although the severity of exacerbations was not increased. ${ }^{18}$ Our study noted a slightly higher number of hospitalizations during this winter period (65 versus 58), but we found a significantly higher number of patients during the April through September study period coded as having major complications and comorbidities, (based on MS-DRG) conflicting with the expected finding. This was corroborated when we analyzed the CMI and found our post-intervention period study group to be higher than that of the pre-intervention group, (1.057 versus 0.993, $p=0.029)$. Our sample size was limited to 123 participants due to the relatively short duration of the study periods, and prospective studies would need a larger number of patients in order to be adequately powered to detect a difference. Despite these limitations, the decreased administrations and overall cost savings per patient make our findings provocative.

\section{Conclusion}

This study demonstrates that the use of a LABA/ LAMA combination inhaler for COPD exacerbation treatment is potentially reasonable. After adjustment for potential confounding variables, the incorporation of UME/VIL was not statistically associated with an increase in 30-day readmission rate. We also did not 
observe significant differences in 30-day mortality or hospital length of stay. Adequately powered, randomized prospective trials are needed to validate whether protocolized use of UME/VIL is a safe and cost-effective alternative to multiple-daily dose regimens used for COPD exacerbation treatment.

\section{Acknowledgements}

The authors thank Shane Webber for his assistance in data extraction and organization from EMR sources. Authors' contributions: TC, GB, TL, BA, and DL participated in study design and concept of the study. Statistical analyses were overseen by DL and TC. All the authors contributed to the acquisition and interpretation of data and also in drafting and revision of the manuscript, and are guarantors of the paper, taking responsibility for the integrity of the work as a whole, from inception to published article.

\section{Declaration of Interest}

None of the authors have any real or apparent conflicts of interest, including no financial or consulting relationships, to disclose. 


\section{References}

1. Bourdet SV, Williams DM. Bourdet SV, et al. Chronic Obstructive Pulmonary Disease. In: DiPiro JT, Talbert RL, Yee GC, Matzke GR, Wells BG, Posey L. Eds. Pharmacotherapy: A Pathophysiologic Approach, 9e. New York, NY: McGraw-Hill; 2014. http://accesspharmacy.mhmedical.com/content.aspx?boo kid $=689 \&$ Sectionid $=48811464$.

Published 2014. Accessed October 17, 2016.

2. Centers for Disease Control and Prevention. Chronic obstructive pulmonary disease among adults - United States, 2011. MMWR Morb Mortal Wkly Rep. 2012; 61(46):938-943.

3. World Health Organization. World health statistics March 2015. World Health Organization website. http://www.who.int/ mediacentre/factsheets/fs315/en/. Accessed October 17, 2016.

4. Seemungal TA, Hurst JR, Wedzicha JA. Exacerbation rate, health status and mortality in COPD - a review of potential interventions. Int J Chron Obstruct Pulmon Dis. 2009; 4: 203-223. doi: https://doi.org/10.2147/COPD.S3385

5. Global Initiative for Chronic Obstructive Lung Disease (GOLD). Global Strategy for the Diagnosis, Management and Prevention of COPD. 2017. GOLD website.

http://goldcopd.org/wp-content/uploads/2016/12/wms-GOLD2017-Pocket-Guide.pdf. Published 2017. Accessed June 5, 2017.

6. Strassels SA, Smith DH, Sullivan SD, Mahajan PS. The costs of treating COPD in the United States. Chest. 2001; 119(2): 344-352. doi: https://doi.org/10.1378/chest.119.2.344

7. Druss BG, Marcus SC, Olfson M, Pincus HA. The most expensive medical conditions in America. Health Aff (Millwood). 2002; 21(4): 105-111. doi: https://doi.org/10.1377/hlthaff.21.4.105

8. Kyriakoudi A, Daganou M, Kyriakopoulou M, et al. COPD exacerbation in ICU. Pneumon. 2014; 27(3):242-248.

9. Centers for Medicare and Medicaid Services (CMS). Readmissions reduction program (HRRP). CMS website. https:// wWw.cms.gov/medicare/medicare-fee-for-service-payment/ acuteinpatientpps/readmissions-reduction-program.html. October 6, 2016.

10. Calzetta L, Rogliani P, Matera MG, Cazzola M. Systematic review with meta-analysis of dual bronchodilation with LAMA/LABA for the treatment of stable COPD. Chest. 2016; 149(5): 1181-1196. doi: https://doi.org/10.1016/j.chest.2016.02.646

11. Tashkin DP, Ferguson GT. Combination bronchodilator therapy in the management of chronic obstructive pulmonary disease. Respir Res. 2013; 14(5): 1-13.

doi: https://doi.org/10.1186/1465-9921-14-49
12. Soler-Cataluna JJ, Martinez-Garcia MA, Sanchez PR, Salcedo E, Navarro M, Ochando R. Severe acute exacerbations and mortality in patients with chronic obstructive pulmonary disease. Thorax. 2005; 60(11): 925-931.

doi: https://doi.org/10.1136/thx.2005.040527

13. Centers for Disease Control and Prevention(CDC). Pneumococcal vaccine timing for adults. CDC website. https:// www.cdc.gov/vaccines/vpd/pneumo/downloads/pneumovaccine-timing.pdf Published November 2015. Accessed March 22, 2017

14. Ramsey SD, Hobbs FDR. Chronic obstructive pulmonary disease, risk factors, and outcome trials: comparisons with cardiovascular disease. Proc Am Thorac Soc. 2006; 3(7):635-640. doi: https://doi.org/10.1513/pats.200603-094SS

15. Centurion VP, Huang F, Naureckas ET, et al. Confirmatory spirometry for adults hospitalized with a diagnosis of asthma or chronic obstructive pulmonary disease exacerbation. BMC Pulm Med. 2012; 12: 73. doi: https://doi.org/10.1186/1471-2466-12-73

16. Hsia DC, Krushat WM, Fagan AB, Tebbutt JA, Kusserow RP. Accuracy of diagnostic coding for Medicare patients under the prospective-payment system. N Engl J Med. 1988; 318: 352-355. doi: https://doi.org/10.1056/NEJM198802113180604

17. Centers for Disease Control and Prevention (CDC). CMS reporting requirements FAQs. CDC website. https://www.cdc. gov/nhsn/PDFs/CMS/faq/FAQs-CMS-Reporting-Requireme nts.pdf. Published 2017. Accessed March 22, 2017

18. Jenkins CR, Celli B, Anderson JA, et al. Seasonality and determinants of moderate and severe COPD exacerbations in the TORCH study. Eur Respir J. 2012; 39(1): 38-45. doi: https://doi.org/10.1183/09031936.00194610 